\section{Multi-site point of care assessment of Abbott ID NOW rapid molecular test for SARS-CoV-2 in a low- prevalence setting}

Sir,

At the time of writing, Australia has one of the lowest SARS-CoV-2 infection rates globally due mostly to closure of the Australian border, and non-pharmaceutical interventions such as physical distancing in conjunction with high rates of diagnostic testing and isolation of positive cases and their contacts. ${ }^{1}$ To date, highly sensitive reversetranscription PCR (RT-PCR) assays performed in clinical laboratories have been the cornerstone of diagnostic testing for SARS-CoV-2. However, depending on the setting, RTPCR results have taken approximately $24-48$ hours to return: this has led to delays in contact tracing and therefore preventable transmission of disease. ${ }^{2}$ Rapid point of care (POC) antigen and molecular tests are generally less sensitive than RT-PCR assays but it has been suggested that increasing test frequency and decreasing test turnaround time can offset lower test sensitivity for effective COVID19 control. ${ }^{3,4}$ To date, few studies have assessed the performance and implementation of rapid molecular POC SARS-CoV-2 testing in a setting with a low prevalence of SARS-CoV-2.

At the time of study initiation, the Xpert Xpress (Cepheid, USA) was the only Therapeutic Goods Administration-listed rapid POC molecular assay in use in Australia. However, there were severe constraints on supply of tests, especially to Australia, necessitating evaluation of other assays. The ID NOW COVID-19 assay (Abbott, USA) is a rapid, instrumentbased molecular isothermal amplification test for the detection of SARS-CoV-2 from oropharyngeal, nasal and nasopharyngeal swabs (NPS). The ID NOW turnaround time for positive results can be as little as 5 minutes with negative results in 13 minutes.

A March 2021 Cochrane review of rapid SARS-CoV-2 tests found that studies were mainly from Europe or North America. Using data from evaluations following the manufacturer's instructions for use (IFU), the average sensitivity of ID NOW was $73.0 \%$ [95\% confidence interval (CI) 66.8$78.4 \%]$ and average specificity $99.7 \%$ (95\% CI $98.7-99.9 \%)$ with a total of only four evaluations with 812 samples and 222 cases. This Cochrane review found that studies of antigen tests were of higher methodological quality compared to studies of molecular tests. ${ }^{5}$

To date, nine peer-reviewed published studies have evaluated performance of ID NOW with positive agreement/ sensitivity varying between $48-94 \%$ compared to RT-PCR. However, only four of these studies have used dry swabs as recommended in the current manufacturer's IFU. The variable performance of the ID NOW may be attributed to the type of swab used, the time of testing post-onset of symptoms and the reference assay used. ${ }^{5,6}$ Knowledge of test specificity is especially important in low-prevalence use; of the eight studies that assessed specificity of the ID NOW, only one tested more than 80 negative samples.
We undertook a prospective study of participants presenting for SARS-CoV-2 testing across two academic hospitals located in Victoria, Australia, during a period of low SARS-CoV-2 prevalence. In Victoria, the COVID-19 pandemic has been characterised by two peaks of transmission: the first occurring between March and April 2020 (maximum 622 active cases) and the second between July and September (maximum 7880 active cases). This study commenced on 2 November 2020 and continued until 4 December 2020 after significant public health interventions had controlled transmission, during which time the 14-day average in metropolitan Melbourne decreased from 20.3 to zero new cases per day. The two participating hospital networks were Monash Health (Clayton and Casey screening clinics, located in Melbourne's south-eastern suburbs) and Austin Hospital screening clinic (located in Melbourne's north-eastern suburbs).

A standard-of-care (SOC) swab for RT-PCR was collected according to local hospital procedure; all sites performed a single combined throat and deep nasal swab, of either one or both nasal cavities. A second-deep nasal swab was collected at the same time using the dry foam swab provided in the ID NOW COVID-19 kit and tested according to the IFU. All swabs were collected by trained healthcare professionals. The Abbott ID NOW COVID-19 test was performed at the point of care.

SOC swabs were collected using either a flocked ESwab (Copan, USA) in $1 \mathrm{~mL}$ liquid amies or flocked swab (Kang Jian, China) in $3 \mathrm{~mL}$ universal transport media and tested using the preferred RT-PCR assay at each of the pilot sites: at Monash Health using the Respiratory Pathogens 12-well assay (AusDiagnostics, Australia) ${ }^{8}$ or Xpert Xpress SARSCoV-2 assay (Cepheid, USA); ${ }^{5}$ at Austin Health using the Coronavirus Typing (8-well) panel (AusDiagnostics) ${ }^{9}$ or the Xpert Xpress SARS-CoV-2 assay.

Statistical analyses were performed using SPSS statistical software package version 27 (SPSS, USA), or GraphPad Prism, version 9.0 (GraphPad, USA).

Ethics review and study approval was provided by Monash Health Human Research and Ethics Committee (RES-200000-678A).

In total, 1044 paired swabs were performed for both ID NOW and RT-PCR testing (634 at Monash Health; 410 at Austin Health) from 1037 participants. One swab was excluded due to missing RT-PCR data leaving 1043 paired swabs for evaluation. Median duration of symptoms was 1 day (range 0-60 days) and median age of participants was 36 years (range 4-91 years); $44.1 \%$ were male and gender information was not reported for three patients. Fifty-five participants were asymptomatic, two had previously confirmed COVID-19, and 986 participants were symptomatic with suspected COVID-19 symptoms.

In this prospective arm of the study the specificity of the Abbott ID NOW test using the kit's dry swab was $99.9 \%$ when tested on 1043 prospectively collected paired swabs (95\% CI 99.47-100\%). The rate of invalid results was $1.9 \%$ on initial testing: four from Monash (where there were 6 users) and 16 from Austin (16 users), all of which were resolved by reinserting the test cartridge. One participant had 
a positive ID NOW result with negative RT-PCR and 1042 participants had negative ID NOW results. Our study includes over double the number of RT-PCR-negative patients included in all currently published studies combined (following the manufacturer's IFU). There were no positive RT-PCR results during the study period (Table 1).

Additionally, patients with recent RT-PCR confirmed SARS-CoV-2 infection (identified through State Health Department notifications) were invited to provide a deep nasal swab collected by a trained healthcare professional using the foam swab provided in the ID NOW COVID-19 kit. A SOC swab was also collected and tested at the Microbiological Diagnostic Unit Public Health Laboratory on the Aptima SARS-CoV-2 assay (Hologic, USA) according to the manufacturer's instructions. For validation purposes, additional PCR-negative patient samples were obtained from testing sites at the University of Melbourne and Monash Health.

Positive agreement amongst the 19 participants with recent RT-PCR-confirmed infection was $76.9 \%(10 / 13)$ for those with less than 7 days since symptom onset compared to Aptima SARS-CoV-2 assay. NPA was 99.7\% (317/318, 95\% CI 98.26-99.99\%) for 318 samples that were negative on Aptima SARS-CoV-2 assay (Table 1). Of the four previous studies that used dry swabs, the sensitivity of the ID NOW was between $48-75 \%$, but these studies were small; the only one of these studies that included more than 31 positive

Table 1 Results for clinical evaluations of ID NOW COVID-19 assay

\begin{tabular}{|c|c|c|}
\hline \multirow[t]{2}{*}{ Retrospective clinical evaluation } & \multicolumn{2}{|c|}{ Days since symptom onset ${ }^{\mathrm{a}}$} \\
\hline & $\begin{array}{l}1-21 \text { days } \\
\text { (median } 3 \text { ) }\end{array}$ & $\begin{array}{l}1-7 \text { days } \\
\text { (median } 2)\end{array}$ \\
\hline Number of positive cases ${ }^{b}$ & 21 & 14 \\
\hline Number of negative cases ${ }^{c}$ & 316 & 314 \\
\hline $\begin{array}{l}\text { Number of positive SARS-CoV-2 samples } \\
\text { by SOC RT-PCR }\end{array}$ & 19 & 13 \\
\hline $\begin{array}{l}\text { Ct range of positive SARS-CoV-2 samples } \\
\text { by SOC RT-PCR }\end{array}$ & $17.1-37.3$ & $17.1-31.8$ \\
\hline $\begin{array}{l}\text { Number of positive SARS-CoV-2 samples } \\
\text { by ID NOW }\end{array}$ & 17 & 11 \\
\hline $\begin{array}{l}\text { Number of false-positive SARS-CoV-2 } \\
\text { samples by ID NOW }\end{array}$ & 1 & 1 \\
\hline $\begin{array}{l}\text { Number of negative SARS-CoV-2 } \\
\text { samples by SOC RT-PCR }\end{array}$ & 318 & 316 \\
\hline $\begin{array}{l}\text { Number of negative SARS-CoV-2 } \\
\text { samples by ID NOW }\end{array}$ & 320 & 317 \\
\hline $\begin{array}{l}\text { Number of false-negative SARS-CoV-2 } \\
\text { samples by ID NOW }\end{array}$ & 3 & 2 \\
\hline Prospective hospital pilot sites & Monash & Austin \\
\hline Staff using test, $n$ & 6 & 16 \\
\hline Total participants, $n$ & 634 & 410 \\
\hline Excluded, $n$ & 0 & 1 \\
\hline Total included, $N$ & 634 & 409 \\
\hline Median age, years (range) & $35(8-83)$ & $36(4-90)$ \\
\hline Male gender, $n(\%)$ & $290(45.7 \%)$ & $167(40.8 \%)$ \\
\hline Asymptomatic, $n(\%)$ & $48(7.6 \%)$ & $7(1.7 \%)$ \\
\hline Median days of symptoms (range) & $2(1-60)$ & $1(0-14)$ \\
\hline Positive RT-PCR result, $n(\%)$ & $0(0 \%)$ & $0(0 \%)$ \\
\hline Positive Abbott ID NOW result, $n(\%)$ & $1(0.2 \%)$ & $0(0 \%)$ \\
\hline
\end{tabular}

${ }^{\text {a }}$ Where known for symptomatic cases. Individuals who were $1-7$ days since symptom onset are a subset of the group of individuals who are 121 days since symptom onset.

${ }^{b}$ Individuals with known COVID-19 infection notified to Department of Health and Human Services (DHHS): only 19 of 21 of these individuals were RT-PCR positive at the time of recruitment to this study.

${ }^{\mathrm{c}}$ Individuals who tested negative for SARS-CoV-2 and were not considered COVID-19 cases by DHHS at the time. patients found a sensitivity of $75 \%$ in 187 RT-PCR positive patients. ${ }^{6}$ In our study, due to the low prevalence of infection during the study period, assessment of clinical sensitivity was limited but consistent with the Cochrane review and with this only other reasonably-sized peer-reviewed publication that followed the manufacturer's IFU. However, further studies are required to assess the effect on sensitivity of using anterior nasal versus deep nasal/NPS.

To assess safety for testing staff, the ability of the Abbott ID NOW sample receiver buffer to inactivate SARS-CoV-2 was studied in three independent experiments. According to the manufacturer's directions, the sample receiver capsules (total $n=10$ ) were pre-heated to $56^{\circ} \mathrm{C}$ for 3 minutes, then $200 \mu \mathrm{L}$ of undiluted SARS-CoV-2 human/Victoria/01/2020 (concentration $10^{5.3} \mathrm{TCID}_{50} / \mathrm{mL}$ ) was pipetted into the $2.5 \mathrm{~mL}$ buffer. As a control, the equivalent volume of room temperature infection media (Minimal Essential Media supplemented with $10 \mu \mathrm{M}$ HEPES, $2 \mathrm{mM}$ glutamine and antibiotics) was also spiked with $200 \mu \mathrm{L}$ SARS-CoV-2. The presence of infectious virus was assessed using a 50\% Tissue Culture Infectious Dose (TCID 50 ) assay. ${ }^{10}$ The preheated buffer was able to reduce the SARSCoV-2 infectious titre to non-detectable levels $\left(<10\right.$ TCID $_{50} /$ $\mathrm{mL}$ ) within 30 seconds of spiking, while infectious virus was recoverable from the spiked infection media control after 5 minutes of incubation (Fig. 1).

One advantage of POC NAT assays over POC antigen tests is result management including objective result interpretation, result recording and potential for automated result communication (such as cloud-based interfacing with laboratory information systems) to enable results communication to patients, their health care providers and public health officials. ${ }^{11}$ We did observe a greater number of invalid results in sites that employed more users, suggesting that experience in running the assay may affect performance. Guidelines for POC testing in Australia recommend training and competency assessments for staff performing testing, in addition to an overarching quality framework to ensure quality control and quality assurance. ${ }^{12}$

In summary, we found this rapid NAT assay to have a high specificity when evaluated prospectively in a real-life low-

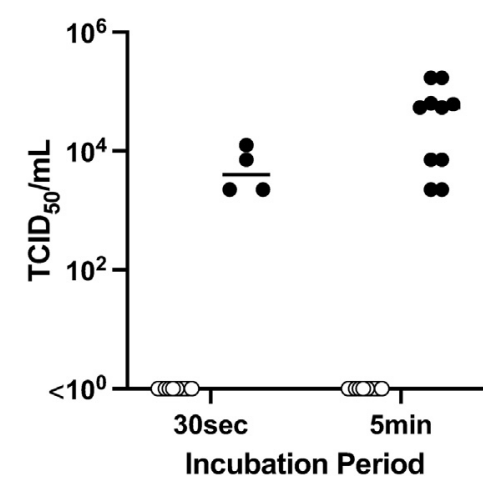

- ID Now

- Infection Media

Fig. 1 Results of virucidal study. When pre-heated to $56^{\circ} \mathrm{C}$ the ID NOW elution/lysis buffer was able to reduce the SARS-CoV-2 infectious titre to nondetectable levels $\left(<10^{0} \mathrm{TCID}_{50} / \mathrm{mL}\right)$ within 30 seconds of spiking, while recovery of infectious virus from the spiked infection media control was between $10^{3.6} \mathrm{TCID}_{50} / \mathrm{mL}$ and $10^{4.92} \mathrm{TCID}_{50} / \mathrm{mL}$ after 5 minutes of incubation $(* * * p<0.001$, two-way ANOVA, Sidak's multiple comparisons test). Data represents pooled results from three independent experiments: Experiment 1 tested $n=3$ ID NOW buffer samples at 30 seconds and at 5 minutes, with $n=3$ spiked infection media at 5 minutes; Experiment 2 was an exact repeat of Experiment 1; Experiment 3 tested $n=4$ ID NOW buffer samples at 30 seconds and at 5 minutes and $n=4$ spiked infection media at 30 seconds and 5 minutes. 
prevalence POC setting. In this low-prevalence context, due to the lower sensitivity when compared to laboratory RTPCR, this and similar rapid POC NAT assays may be most useful in enabling the rapid triage of public health and hospital resources while expediting confirmatory PCR testing.

Acknowledgements: We thank the COVID-19 Diagnostics Research Group, which in addition to the listed authors, also comprises Nick Tayler, Jason A. Trubiano, Olivia Smibert, George Drewett, Fiona James, Socheata Chea, Steven Edwards, Nicole Isles, Michelle Sait and Beau Carr, for their laboratory and clinical support for this study. We also thank Kirsten Holden and staff at Clayton and Casey Screening Clinic, as well as Grace Gibney and staff at Austin Health Screening Clinic, for collecting the extra study swabs from patients.

Conflicts of interest and sources of funding: This research was funded by the Medical Research Future Fund (MRFF) 2020 COVID-19 Diagnostics Grant Opportunity as part of the COVID-19 Strategic Planning and Delivery of Testing program and the Victorian Government Department of Health as part of the Doherty Institute Innovative Testing Program. The funders were not involved in data analysis or manuscript preparation. The authors state that there are no conflicts of interest to disclose.

\section{Maryza Graham ${ }^{1,2, *}$, Stephen Muhi ${ }^{1,3,4, *}$, Tuyet Hoang ${ }^{1}$, Susan A. Ballard ${ }^{1}$, Julie McAuley ${ }^{3}$, Jason C. Kwong, ${ }^{3,5}$, Deborah A. Williamson ${ }^{1,3,6, \dagger}$, Benjamin P. Howden ${ }^{1,3,5, \dagger}$, and the COVID-19 Diagnostics Research Group}

\begin{abstract}
${ }^{1}$ Microbiological Diagnostic Unit Public Health Laboratory, The University of Melbourne at the Peter Doherty Institute for Infection and Immunity, Melbourne, Vic, Australia; ${ }^{2}$ Department of Microbiology and Infectious Diseases, Monash Health, Clayton, Vic, Australia; ${ }^{3}$ Department of Microbiology and Immunology, The University of Melbourne at the Peter Doherty Institute for Infection and Immunity, Melbourne, Vic, Australia; ${ }^{4}$ Victorian Infectious Diseases Service, Royal Melbourne Hospital, Parkville, Vic, Australia; ${ }^{5}$ Department of Infectious Diseases, Austin Hospital, Heidelberg, Vic, Australia; ${ }^{6}$ Department of Microbiology, Royal Melbourne Hospital, Melbourne, Vic, Australia; *十these authors contributed equally; $\ddagger$ COVID-19 Diagnostics Research Group members are listed in the Acknowledgements
\end{abstract}

Contact: Dr Maryza Graham.

E-mail: maryza.graham@monashhealth.org

1. Chang SL, Harding N, Zachreson C, et al. Modelling transmission and control of the COVID-19 pandemic in Australia. Nat Commun 2020; 11: 5710.

2. Kretzschmar ME, Rozhnova G, Bootsma MC, van Boven M, van de Wijgert JH, Bonten MJ. Impact of delays on effectiveness of contact tracing strategies for COVID-19: a modelling study. Lancet Public Health 2020; 5: e452-9.

3. Mina MJ, Parker R, Larremore DB. Rethinking COVID-19 test sensitivity - a strategy for containment. N Engl J Med 2020; 383: e120.

4. Larremore DB, Wilder B, Lester E, et al. Test sensitivity is secondary to frequency and turnaround time for COVID-19 surveillance. medRxiv 2020; 8 Sep: https://doi.org/10.1101/2020.06.22.20136309.

5. Dinnes J, Deeks JJ, Berhane S, et al. Cochrane COVID-19 Diagnostic Test Accuracy Group. Rapid, point-of-care antigen and molecular-based tests for diagnosis of SARS-CoV-2 infection. Cochrane Database Syst Rev 2021; 3: CD013705.
6. Serei VD, Cristelli R, Joho K, et al. Comparison of Abbott ID NOW COVID-19 rapid molecular assay to Cepheid Xpert Xpress SARS-CoV2 assay in dry nasal swabs. Diagn Microbiol Infect Dis 2020; 99: 115208

7. Harrington A, Cox B, Snowdon J, et al. Comparison of Abbott ID Now and Abbott m2000 methods for the detection of SARS-CoV-2 from nasopharyngeal and nasal swabs from symptomatic patients. J Clin Microbiol 2020; 58: e00798-20.

8. Attwood LO, Francis MJ, Hamblin J, Korman TM, Druce J, Graham M. Clinical evaluation of AusDiagnostics SARS-CoV-2 multiplex tandem PCR assay. J Clin Virol 2020; 128: 104448.

9. Williams E, Bond $\mathrm{K}$, Chong B, et al. Implementation and evaluation of a novel real-time multiplex assay for SARS-CoV-2: in-field learnings from a clinical microbiology laboratory. Pathology 2020; 52: 754-9.

10. Lee JYH, Best N, McAuley J, et al. Validation of a single-step, singletube reverse transcription loop-mediated isothermal amplification assay for rapid detection of SARS-CoV-2 RNA. J Med Microbiol 2020; 69 $1169-78$.

11. Muhi S, Tayler N, Hoang T, et al. Multi-site assessment of rapid, pointof-care antigen testing for the diagnosis of SARS-CoV-2 infection in a low-prevalence setting: a validation and implementation study. Lancet Reg Health West Pac 2021; 9: 100115.

12. National Pathology Accreditation Advisory Council. Guidelines for Point of Care Testing (PoCT): (First Edition 2015). Canberra: Australian Government Department of Health, 2015. https://www1. health.gov.au/internet/main/publishing.nsf/Content/health-npaacpoctguid

DOI: https://doi.org/10.1016/j.pathol.2021.07.002

\section{Prolonged PCR positivity in elderly patients infected with SARS-CoV-2}

Sir,

The global coronavirus disease 2019 (COVID-19) pandemic has raised many questions around the transmission dynamics of severe acute respiratory syndrome coronavirus 2 (SARSCoV-2). Among these, defining the period of infectivity and, critically, proving clearance of the virus from the respiratory tract are paramount to public health efforts.

Reverse transcriptase polymerase chain reaction (RT-PCR) assays are now commonplace in diagnostic laboratories, and their limitations are well recognised. Clinicians are aware that common acute respiratory viral infections may be followed by prolonged upper respiratory tract PCR positivity. PCR assays specifically detect viral genetic material but typically cannot distinguish between intact replication-competent virus and residual, non-viable viral fragments. Recent studies have demonstrated that SARS-CoV-2 RNA may be detected well beyond the period in which viable virus can be cultured. ${ }^{1-3}$ Shedding of culturable SARS-CoV-2 has been seen up to a median of 9 days from illness onset, ${ }^{3}$ with PCR positivity persisting for a median of 17 days, longer in the upper respiratory tract than in the lower airway. ${ }^{2}$ In our own centre, viable virus is more likely to be isolated in patients with more severe disease and with lower PCR cycle threshold $(\mathrm{Ct})$ values, with no virus cultured from respiratory samples with a $\mathrm{Ct}>32$.

All respiratory tract samples received for SARS-CoV-2 reverse transcriptase polymerase chain reaction (RT-PCR) testing at the Centre for Infectious Diseases and Microbiology Laboratory Services, NSW Health Pathology - Institute of Clinical Pathology and Medical Research (ICPMR), Westmead, Australia, from 22 January to 30 June 2020 were assessed. Only individuals whose samples were positive for SARS-CoV-2 RNA by RT-PCR testing more than 14 days 